\title{
The accuracy of the Ishii score chart in predicting sarcopenia in the elderly community in Chengdu
}

\author{
Xiaoyan Chen', Lisha Hou', Ying Zhang ${ }^{2}$, Shuyue Luo ${ }^{2}$ and Birong Dong ${ }^{2 *}$
}

\begin{abstract}
Background: Sarcopenia is a disorder associated with age that reduces the mass of skeletal muscles, the strength of muscles, and/or physical activity. It increases the risk of fall incidence which can result in fractures, hospitalizations, limited movement, and considerably decreased quality of life. Hence, it is needed to explore candidate screening tools to evaluate sarcopenia in the initial phases. The reported studies have been revealed that the sensitivity and specificity of the Ishii score chart are higher. However, the Ishii score chart is principally based on the European Working Group on Sarcopenia in Older People (EWGSOP) consensus. Recently, the Asian Working Group for Sarcopenia (AWGS) 2019 consensus has updated its diagnostic criteria for sarcopenia, which was previously similar to the EWGSOP. Hence, it is necessary to determine whether the Ishii score chart is appropriate for use among the elderly population in China. The current study aimed to validate the precision of the Ishii score chart, within the Chinese old aged community to establish an effective model for the evaluation of sarcopenia.
\end{abstract}

Methods: The AWGS2019 sarcopenia diagnostic criteria were used as a standard, and among the elderly community, the accuracy of the Ishii score chart was determined by using indicators, including specificity, sensitivity, negative and positive predictive values, negative and positive likelihood ratios, Youden index, and receiver operating characteristic $(\mathrm{ROC})$ curve.

Results: In the elderly Chengdu community, the prevalence rate of sarcopenia was $18.38 \%, 19.91 \%$ for males and $16.91 \%$ for females. The Ishii score chart predicts sarcopenia at an AUC value of 0.84 with $95 \%$ confidence interval $(\mathrm{Cl})$, ranging between 0.80 and 0.89 for females, and at an AUC value of 0.81 with $95 \%$ $\mathrm{Cl}$, ranging between 0.75 and 0.86 for males.According to the original cut-off, which was set at 120 points for females, the corresponding sensitivity was $46.91 \%$ and the specificity was $93.22 \%$. The 105 cut-off points (original) set for males revealed a corresponding sensitivity of $64.94 \%$ and the specificity of $85.46 \%$. However, the original cut-off value exhibited low sensitivity, hence, we selected a new cut-off value. With the new cutoff value, the sensitivity, specificity, positive and negative predictive values for sarcopenia were $75.31 \%$, $79.9 \%, 43 \%$, and $94 \%$ for females, and $70.65 \%, 81.35 \%, 49 \%$, and $92 \%$ for males, respectively.

(Continued on next page)

\footnotetext{
* Correspondence: birongdong123@outlook.com

${ }^{2}$ National Clinical Research Center for Geriatrics, West China Hospital, Sichuan University, No. 37, Guo Xue Xiang Renmin Nan Lu Chengdu, Sichuan, China

Full list of author information is available at the end of the article
}

C C The Author(s). 2021 Open Access This article is licensed under a Creative Commons Attribution 4.0 International License, which permits use, sharing, adaptation, distribution and reproduction in any medium or format, as long as you give appropriate credit to the original author(s) and the source, provide a link to the Creative Commons licence, and indicate if changes were made. The images or other third party material in this article are included in the article's Creative Commons licence, unless indicated otherwise in a credit line to the material. If material is not included in the article's Creative Commons licence and your intended use is not permitted by statutory regulation or exceeds the permitted use, you will need to obtain permission directly from the copyright holder. To view a copy of this licence, visit http://creativecommons.org/licenses/by/4.0/. The Creative Commons Public Domain Dedication waiver (http://creativecommons.org/publicdomain/zero/1.0/) applies to the data made available in this article, unless otherwise stated in a credit line to the data. 
(Continued from previous page)

Conclusions: The Ishii score chart was used for the prediction of sarcopenia in the old-age people of the Chengdu community and the obtained results showed a high value of predictability. Hence, more than 95 and 102 points were suggested for males and females, accordingly which can set to be the diagnostic cut-off values for the prediction of sarcopenia.

Keywords: Sarcopenia, Elderly, Community, AWGS

\section{Background}

Sarcopenia is an age-associated disorder characterized by weakness of skeletal muscle strength and gradual loss of muscle mass, which may lead to the inability of physical performance [1]. This disorder badly affects the quality of life in several ways including high chances of fractures due to falling, the burden of their body weight, and subsequently a loss of mobility [2]. The underlined disorder, in the beginning, has no obvious symptoms. However, after a severe disability or fall, it becomes appear [3]. In view of these facts, it is necessary to develop significant screening strategies that can reveal the disease in the initial phases and help the elder patients within the community. At present, for sarcopenia, the frequently used screening tools are the SARC-F scale (muscle strength, assisted walking, ability to rise from chair, ability to climb stairs, and incidence of falls) [4], calf circumference (CC) [5], and the Ishii score chart [6], etc. An ideal screening model should define a reasonable cut-off point, be effective, reliable, convenient, economical, and have reasonable accuracy, sensitivity, and specificity [7]. In multiple countries, SARC-F is considered to be the most significant and commonly used tool for the screening purpose of sarcopenia. SARC-F has four subjective questions and one objective question on the screening questionnaire, the results of which may be affected by an elderly individual's life attitude and psychology [8]. In this view, the specificity of SARC-F remains higher, and the sensitivity is lower [9, 10]. In 2019, CC was recommended by AWGS as an effective tool for the screening of sarcopenia [1]. The underlined recommendations were made on the basis of Japanese research, reported by Kawakami $\mathrm{R}$ et al. where calf circumference measurement was compared with dual $x$-ray absorptiometry (DXA) to predict the onset of sarcopenia as being $34 \mathrm{~cm}$ and below in males (sensitivity $88 \%$, specificity $91 \%$ ) and $33 \mathrm{~cm}$ and below in females (sensitivity $76 \%$, specificity $73 \%)$ [11]. Conversely, in another study, Yves Rolland et al. retrospectively analyzed 1458 French females over the age of 70 with no history of hip fracture and found that the calf circumference was associated with skeletal muscle mass, but not sarcopenia (defined by DXA) [12]. In this study, we compared the calf circumference measurement with appendicular skeletal muscle mass (ASM) estimated by bioelectrical impedance analysis (BIA), and predicted the sarcopenia onset to be $34 \mathrm{~cm}$ and below in males (sensitivity $80.5 \%$, specificity $55.4 \%$ ) and $33 \mathrm{~cm}$ and below in females (sensitivity $70.9 \%$, specificity $77.8 \%$ ). The significance of calf circumference in sarcopenia screening is controversial. On the basis of EWGSOP consensus, the Ishii score chart was initially constructed by Ishii and his colleagues. This screening tool used inputs like age (year), grip strength $(\mathrm{kg})$, and $\mathrm{CC}(\mathrm{cm})$ as objective signs that were put into a mathematical calculation to infer the incidence of sarcopenia. The male score, for example, was calculated as follows: $0.62 \times($ age-64) $-3.09 \times$ (grip strength -50$)-4.64 \times($ CC-42). Moreover, according to Ishii et al. $\geq 105$ points was the diagnostic cut-off for sarcopenia in males [6]. Based on this, at a cut-off value of 105 in males, the sensitivity, specificity, positive, and negative predictive values for sarcopenia were $84.9 \%$, $88.2 \%, 54.4 \%$, and $97.5 \%$. Meanwhile, the female score was calculated as follows: $0.8 \times$ (age64) $-5.09 \times($ grip strength -34$)-3.28 \times($ CC-42). Moreover, Ishii et al. recommended $\geq 120$ points to be the diagnostic cut-off for sarcopenia in females [6]. Based on this, at a cut-off value of 120 in females, the sensitivity, specificity, positive, and negative predictive values for sarcopenia were $75.5 \%, 92.0 \%, 72.8 \%$, and $93 \%$ respectively. More recently, Li Min [13] et al. employed the AWGS2014 consensus as the diagnostic criteria for sarcopenia to verify the accuracy of the Ishii score chart in predicting sarcopenia. They found that the sensitivity and specificity of males were 90.9 and $70.4 \%$, respectively. In addition, the sensitivity and specificity of females were 82.4 and $70 \%$, respectively. Therefore, the Ishii score chart predicts sarcopenia with high sensitivity and specificity.

Since the AWGS2019 consensus updated its prior diagnostic measures, whether the Ishii score chart is considerable for the old age Chinese population or needs further verification.This study, therefore, intends to validate the accuracy of the Ishii score chart in screening sarcopenia among the elderly Chinese population in an effort to establish a reliable and efficient model for sarcopenia screening. 


\section{Method}

\section{Participants}

In 2014, a total of 941 elderly people with ages ranging from 60 to 92 were chosen from the Yulin, Jumper Tower, and Grout Street communities. These peoples (recruited for the current study) were living there for more than a year. They were informed by posting advertisements in the local community and by word-of-mouth by family doctors in WeChat groups. Prior to study participation, subjects signed informed consent (the participants who were unable to write issued an authorization letter to their legal guardians by providing a thumb print and the authorization letter were also signed by the legal guardians. After being authorized, the legal guardians proceeded to provide written consent on behalf of the participants and the participants then assented by providing a thumb print), and their approval was provided by the Institutional Review Committee/ Independent Ethics Committee (IRB/ IEC). Herein, the elder people with severe disorder or cancer at an advanced stage, surgical injuries to the leg/foot or hand/wrist in the last 90 days, any kind of physical disability, electronic devices, orthopedic metal implants, and those using diuretics (except for the ones who used diuretics against hypertension and the stability of dose occurs for more than 21 days), or any other disease or condition which was not considered appropriate by the investigator were excluded.

\section{Muscle mass measurement}

Muscle mass was assessed using BIA and an Inbody 720 (Inbody720, Biospace China Inc.). ASM was defined as the skeletal muscle present in the arms and legs. The values of ASM were normalized by height in metersquared for obtaining appendicular skeletal muscle mass index (ASMI) $(\mathrm{kg} / \mathrm{m} 2)[1]$.

\section{Muscle strength measurement}

Handgrip strength was used for evaluating the strength of muscles by a digital grip strength dynamometer (CAMRY EH 101), followed by conducting measurements on each hand $(n=3)$, and then the maximum values were examined.

\section{Physical performance measurement}

Physical performance was evaluated by the typical gait speed $(6 \mathrm{~m})$, suitable to $0.01 \mathrm{~s}$. For about $5 \mathrm{~min}$, participants were indorsed to warm up and $6 \mathrm{~m}$ walk (twice) was performed, followed by recording and analyzing the average speed.

\section{Other measurements}

The Tsinghua Tongfang Height and Weight Tester were used to measure the height and weight of patients. This instrument is commonly used for physical examinations in clinical practices. The measurement of height was carried out in $\mathrm{cm}$ (accurate to $0.1 \mathrm{~cm}$ ), followed by measuring the weight in $\mathrm{kg}$ (accurate to $0.01 \mathrm{~kg}$ ) and the $\mathrm{CC}$ in $\mathrm{cm}(0.1 \mathrm{~cm})$. The measurement of these indices was carried out two times, followed by analyzing the average values.

\section{Sarcopenia classification and measurement of each component of sarcopenia}

In this study, the suggestions of the AWGS2019 were followed [1]. A low muscle mass and low strength of the muscle or low physical activities are needed by proposed diagnostic criteria. The cut-off value of ASMI was $7.0 \mathrm{~kg} / \mathrm{m}^{2}$ and $5.7 \mathrm{~kg} / \mathrm{m}^{2}$ in males and females, accordingly, followed by recording a grip strength of $<18 \mathrm{~kg}$ and $<28 \mathrm{~kg}$ for females and males, accordingly. A gait speed of $<1.0 \mathrm{~m} / \mathrm{s}$ represents a low level of physical performance.

\section{Statistical analysis}

The stratification of statistical analysis was carried out by sex. IBM SPSS 21.0 (IBM, Armonk, NY, USA) was employed for conducting statistical analysis. A twosided p less than 0.05 was regarded as statistically considerable. In this study, Constant data was concluded as mean $\pm \mathrm{SD}$ or median with IQR, which is dependent on the distribution of data. Categorical variables were reported as numbers (percentage). Statistical analyses between baseline characteristics were performed using the Rank-sum, Student's t-test, and Pearson's chisquare test. The area under the ROC curve (AUC ) was used for evaluating the precision of the Ishii score chart in expecting sarcopenia. The AUC was not affected by population prevalence and was an ideal comprehensive index used to determine the precision of the screening tool. AUC was usually between 0.5 and 1 . Moreover, the closer the AUC value was to 1, the higher was the diagnostic value of the tool. To evaluate the accuracy of the original cut-off value of the Ishii score chart for sarcopenia prediction, we assessed the following variables: specificity, sensitivity, positive and negative predictive value, as well as positive and negative likelihood ratios (LR). Furthermore, to evaluate the accuracy of the updated AWGS2019 sarcopenia diagnostic criteria, we selected a new cut-off point for predicting sarcopenia, one that maximized the Youden index. Among the variables analyzed, a positive predictive value referred to the proportion of standard-diagnosed disease cases (true positives) among subjects with positive diagnostic tests. Alternately, a negative predictive value referred to the portion of the standard-diagnosed non-disease cases (true negatives), among the negative diagnostic test subjects. The LR reflected authenticity and 
composed of a composite index representing sensitivity and specificity. In other words, the ratio referred to the probability of getting a test result from subjects with the condition/disease to the probability of getting the test result from subjects without the condition/disease. A positive LR represented the probability of accurately determining positive diagnosis and was calculated as a multiple of the probability of wrongly judging positive. Therefore, a highly positive LR represented a high diagnostic value of the screening tool. Conversely, a negative LR referred to the probability of a false negative and was calculated as a multiple of the probability of a true negative. In this case, a smaller negative likelihood ratio represented a higher diagnostic value. Therefore, a positive and negative LR was $>10$ and $<0.1$, accordingly. And the possibility of diagnosis or exclusion of disease was significantly elevated. Lastly, the Youden index was calculated as the sum of the specificity and sensitivity of the diagnostic trial minus the base value of 1 (or $100 \%$ ) and represented the overall ability of the diagnostic tool to detect true positives and true negatives. The higher the Youden index value, the higher the overall ability of the screening tool to detect true patients versus non-patients.

\section{Result}

In 2014, a total of 941 people with ages ranging from 60 to 92 were chosen from the Yulin, Jumper Tower, and Grout Street communities.The prevalence rate of sarcopenia was $18.38 \%, 19.91 \%$ for males and $16.91 \%$ for females, the various characteristics of participants by gender are listed in Table 1.

Our results revealed that the Ishii score chart predicted sarcopenia in females when the AUC was 0.84 with $95 \%$ confidence interval $(\mathrm{CI})$, ranging from 0.80 to 0.89 , and predicted sarcopenia in males when the AUC was 0.81 with $95 \% \mathrm{CI}$, ranging from 0.75 to 0.86 , as illustrated in Figs. 1 and 2.

The original cut-off point for females was 120, recommended by the Ishii score chart. The corresponding sensitivity was $46.91 \%$ and the specificity was $93.22 \%$. The positive predictive value was $58 \%$, the negative predictive value was $90 \%$, the positive LR was 6.92 , and the negative LR was 0.57 , as shown in Table 2 . The original cut-off point for males was 105 , recommended by the Ishii score chart. The corresponding sensitivity, specificity, positive and negative predictive values, positive and negative LR values were $64.94 \%, 85.46 \%, 47 \%, 92 \%$, 4.47, and 0.41, respectively, as shown in Table 2.

The AUC of the Ishii score chart was greater than 0.8 which leads to the identification of sarcopenia in males and females. Regarding the high predictive value, the underlined model can be used for the screening of sarcopenia in the Chinese elderly population. However, the sensitivity of the original cut-off value is low when applied to the population and required further adjustment. We, therefore, set a new cut-off value.In females, at the highest Youden index value of 102, the sensitivity and specificity of predictability were 75.31 and $79.9 \%$, respectively, the positive and negative predictive values were 43 and $94 \%$, respectively, the positive and negative LR were 3.75 and 0.31 , respectively (Table 2). In males, the sensitivity and specificity of predicting sarcopenia were 70.65 and $81.35 \%$ when the Youden index reached the highest score of 95, the positive and negative predictive, positive and negative LR values were $49 \%, 92 \%$, 3.79 , and 0.36 , respectively (Table 2 ).

Table 1 The characteristics of participants

\begin{tabular}{|c|c|c|c|c|}
\hline Variable & Total $(N=941)$ & Male $(N=462)$ & Female $(N=479)$ & $P$-value \\
\hline Age (years), $n(\%)$ & & & & 0.186 \\
\hline$<65$ & $306(32.52)$ & $146(31.60)$ & $160(33.40)$ & \\
\hline $65-69$ & $240(25.50)$ & $110(23.81)$ & $130(27.14)$ & \\
\hline $70-79$ & $344(36.56)$ & $181(39.18)$ & $163(34.03)$ & \\
\hline$\geq 80$ & $51(5.42)$ & $25(5.41)$ & $26(5.43)$ & \\
\hline Height (m) & $1.58 \pm 0.84$ & $1.64 \pm 0.06$ & $1.53 \pm 0.06$ & $<0.001$ \\
\hline ASMI (kg/m2) & $6.65 \pm 1.25$ & $6.95 \pm 1.14$ & $6.36 \pm 1.27$ & $<0.001$ \\
\hline Grip strength (kg) & $29.15 \pm 8.96$ & $35.66 \pm 7.52$ & $22.88 \pm 4.79$ & $<0.001$ \\
\hline Gait speed (m/s) & $1.02 \pm 0.17$ & $1.08 \pm 0.20$ & $1.02 \pm 0.17$ & $<0.001$ \\
\hline Calf circumference $(\mathrm{cm})$ & $34.75 \pm 3.00$ & $35.54 \pm 2.92$ & $33.99 \pm 2.89$ & $<0.001$ \\
\hline Sarcopenia, n(\%) & $173(18.38)$ & $92(19.91)$ & 81 (16.91) & 0.234 \\
\hline
\end{tabular}




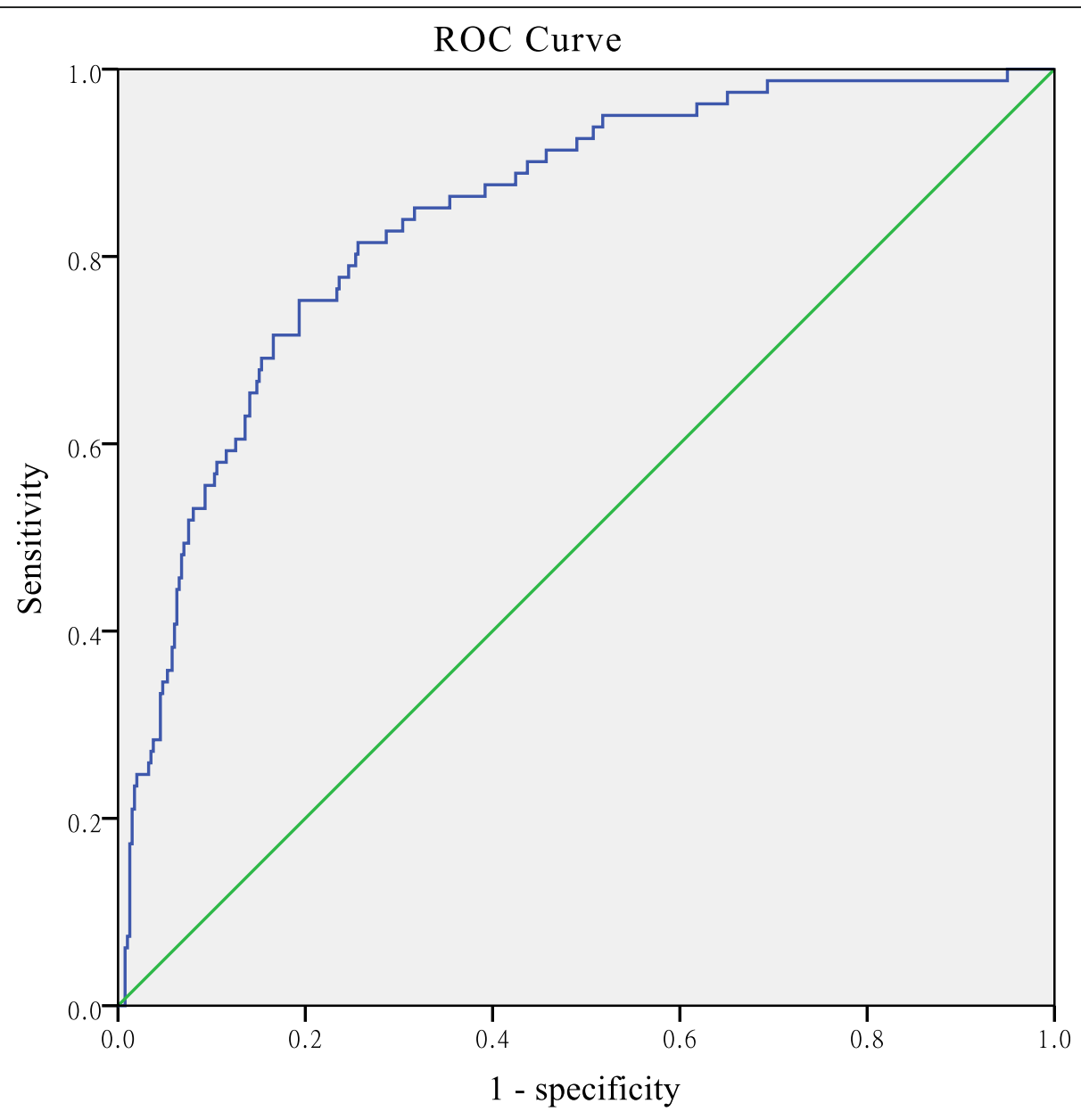

Fig. 1 The Ishii score chart predicting sarcopenia in the female elderly community. AUC was 0.84 (95\% Cl:0.80-0.89)

\section{Discussion}

In this study, the Ishii score chart was used for predicting sarcopenia (in males and females), and the obtained results revealed that the value of AUC was greater than 0.8 which shows the high value of prediction. Hence, the high predicted value of the Ishii score chart was considered as predictability of sarcopenia.

Commonly, the sensitivity and negative estimated values should be higher of the "rule-out" screening test, while the specificity and positive estimated values should be higher of the "rule-in" screening test [14]. In recent decades, screening methods, such as "rule- out" tests have been employed to determine people who are not at risk of sarcopenia. Therefore, our focus should be on the sensitivity and negative predictive values of the screening model. In the current study, the Ishii score chart was used for predicting sarcopenia, and the obtained results revealed that in males and females, the negative predictive values were 92 and $90 \%$, accordingly which suggested that the Ishii score chart has an elevated value of negative prediction. Moreover, in the Ishii model, the sensitivity of the original cut-off value has lower sensitivity. Generally, the sensitivity values are based on the cut-off point, which revealed that variations can occur in the values of sensitivity in accordance with the alterations in the cut-off point. Therefore, a new cut-off value was selected to increase the sensitivity of the prediction. As expected, with the new cut-off value, the Ishii scale sensitivity (in females) increased from 46.91 to $75.31 \%$, and the Ishii scale sensitivity (in males) in predicting sarcopenia increased from 64.94 to $70.65 \%$.

The current study has some limitations. First, the verification of the Ishii score chart was carried out by using selected communities in Chengdu, while in other regions, the populations were not verified. 


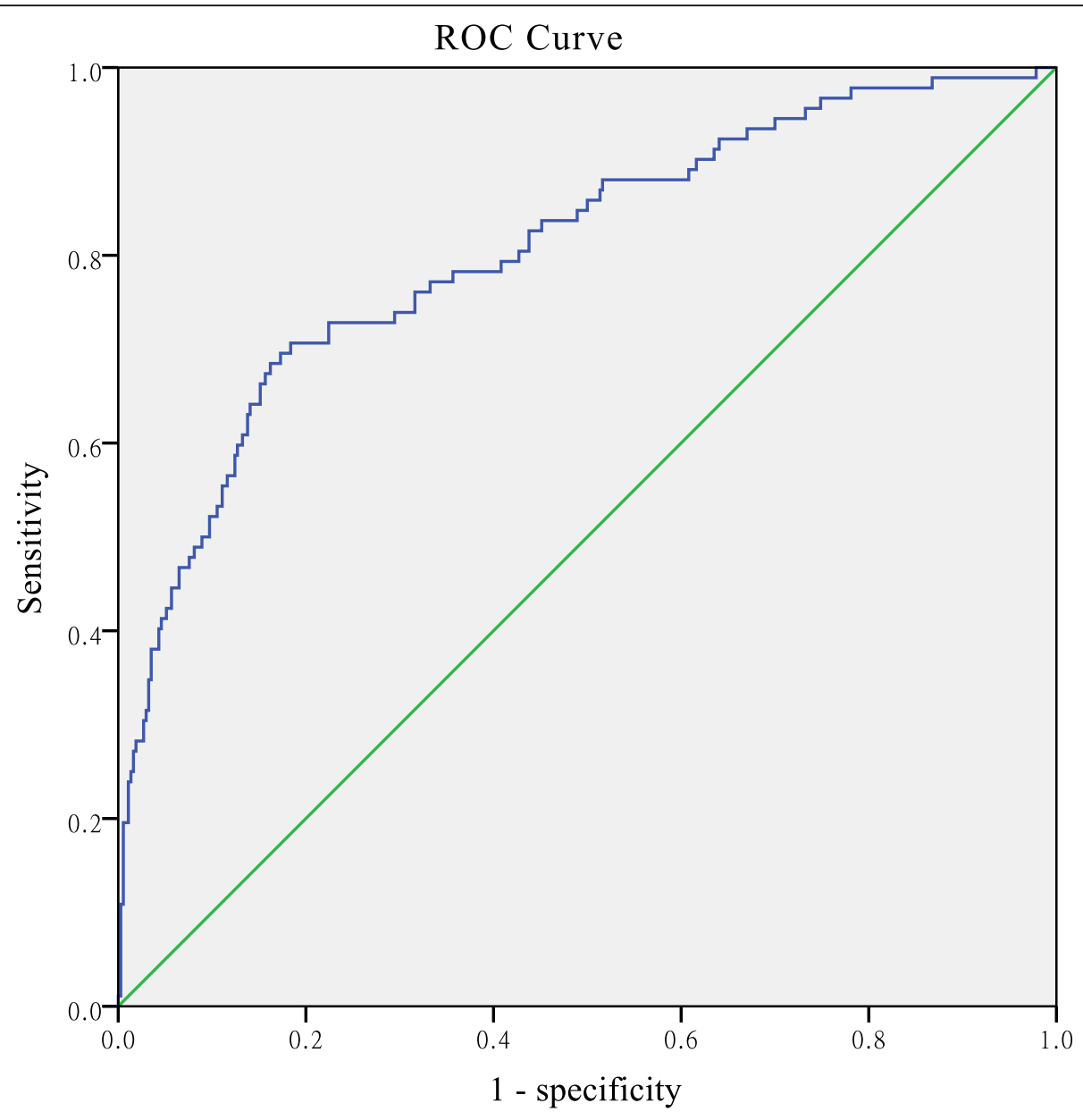

Fig. 2 The Ishii score chart predicting sarcopenia in the male elderly community. AUC was 0.81 (95\% Cl: 0.75-0.86)

Hence, there is no clarity that whether the underlined data can be used for populations in other regions or countries. Second, all participants in this study were healthy volunteers, which may have introduced a deviation in the involved population. Therefore, further studies are needed by using a population with large sample size.

\section{Conclusions}

The Ishii score chart was used for the prediction of sarcopenia in the old-age people of the Chengdu community and the obtained results showed a high value of predictability. Hence, more than 95 and 102 points were suggested for males and females, accordingly which can set to be the diagnostic cut-off values for the prediction of sarcopenia.

Table 2 The accuracy of the Ishii score chart in predicting sarcopenia

\begin{tabular}{|c|c|c|c|c|c|c|c|}
\hline Model & & Sensitivity & Specificity & $\begin{array}{l}\text { Positive } \\
\text { predictive } \\
\text { values }\end{array}$ & $\begin{array}{l}\text { Negative } \\
\text { predictive } \\
\text { values }\end{array}$ & $\begin{array}{l}\text { Positive } \\
\text { likelihood } \\
\text { ratio }\end{array}$ & $\begin{array}{l}\text { Negative } \\
\text { likelihood } \\
\text { ratio }\end{array}$ \\
\hline \multicolumn{8}{|l|}{ Male } \\
\hline \multirow[t]{2}{*}{ sarcopenia } & original cut-off & $64.94 \%$ & $85.46 \%$ & $47 \%$ & $92 \%$ & 4.47 & 0.41 \\
\hline & new cut-off & $70.65 \%$ & $81.35 \%$ & $49 \%$ & $92 \%$ & 3.79 & 0.36 \\
\hline \multicolumn{8}{|l|}{ Female } \\
\hline \multirow[t]{2}{*}{ sarcopenia } & original cut-off & $46.91 \%$ & $93.22 \%$ & $58 \%$ & $90 \%$ & 6.92 & 0.57 \\
\hline & new cut-off & $75.31 \%$ & $79.9 \%$ & $43 \%$ & $94 \%$ & 3.75 & 0.31 \\
\hline
\end{tabular}

Sarcopenia: the presence of low muscle mass plus the presence of either low muscle strength or low physical performance. The original cut-off point was recommended by the Ishii score chart: 120 for females and 105 for males; The new cut-off was the highest score of the Youden index:102 for females and 95 for males 


\section{Abbreviations}

AWGS: Asian Working Group for Sarcopenia; ASMI: Appendicular skeletal muscle mass index; ASM: Appendicular skeletal muscle mass; AUC: Area under the curve; BIA: Bioelectrical impedance analysis; CC: Calf circumference; Cl: Confidence interval; DXA: Dual x-ray absorptiometry; EWGSOP: European Working Group on Sarcopenia in Older People; LR: Likelihood ratio; ROC: Receiver operating characteristic; SARC-F: Strength Assistance with walking, Rise from a chair, climb stairs, and Falls scale

\section{Acknowledgements}

We thank the Yulin Community Health Center, Chengdu who supported the recruitment of all study participants.

\section{Authors' contributions}

$X C$ designed the study and wrote the paper. $L H, Y Z$, and SL take part in the interpretation of data. BD reviewed and edited the manuscript. All authors have read and approved the manuscript.

\section{Funding}

This work was supported by Platform Construction of National Clinical Research Center for Geriatric Medicine (Supported by 1.3.5 project for disciplines of excellence, West China Hospital, Sichuan University). Item Number: ZY2017201. The funding body had no role in the design of the study and collection, analysis, interpretation of data or in writing the manuscript.

\section{Availability of data and materials}

The datasets generated and analyzed during the current study are not publicly available due to this is a database which has a lot of important information and we are applying some important projects based on this. But this datasets will be available 2 years later and is also available now from the corresponding author on a reasonable request.

\section{Declarations}

Ethics approval and consent to participate

This study was approved by the Ethics Committee of West China Hospital, Sichuan University. Participants were recruited by advertising and asked verbally by the researchers about their willingness to take part in the study. Before investigation, informed consent was signed and obtained by each participant(the participants who were unable to write issued an authorization letter to their legal guardians by providing a thumb print and the authorization letter were also signed by the legal guardians. After being authorized, the legal guardians proceeded to provide written consent on behalf of the participants and the participants then assented by providing a thumb print).

\section{Consent for publication}

Not applicable.

\section{Competing interests}

The authors declare no competing interests.

\section{Author details}

${ }^{1}$ Zigong Mental Health Center, Zigong, Sichuan, China. ${ }^{2}$ National Clinical Research Center for Geriatrics, West China Hospital, Sichuan University, No. 37, Guo Xue Xiang Renmin Nan Lu Chengdu, Sichuan, China.

\section{Received: 23 October 2020 Accepted: 27 April 2021}

Published online: 08 May 2021

\section{References}

1. Chen LK, Woo J, Assantachai P, et al. Asian Working Group for Sarcopenia: 2019 consensus update on sarcopenia diagnosis and treatment. J Am Med Dir Assoc. 2020;21(3):300-307. https://doi.org/10.1016/j.jamda. 2019.12.012.

2. Cruz-Jentoft AJ, Sayer AA. Sarcopenia. Lancet. 2019;393(10191):2636-46.

3. Visvanathan R, Chapman I. Preventing sarcopaenia in older people. Maturitas. 2010;66(4):383-8.

4. Malmstrom TK, Morley JE. SARC-F: a simple questionnaire to rapidly diagnose sarcopenia. J Am Med Dir Assoc. 2013;14(8):531-2.
5. Barbosa-Silva TG, Menezes AMB, Bielemann RM, et al. Enhancing SARC-F: improving sarcopenia screening in the clinical practice. J Am Med Dir Assoc. 2016;17(12):1136-41.

6. Ishii S, Tanaka T, Shibasaki K, et al. Development of a simple screening test for sarcopenia in older adults. Geriatr Gerontol Int. 2014;14(Suppl 1):93-101.

7. Grimes DA, Schulz KF. Uses and abuses of screening tests. Lancet. 2002: 359(9309):881-4.

8. Cao L, Chen S, Zou C, et al. A pilot study of the SARC-F scale on screening sarcopenia and physical disability in the Chinese older people. J Nutr Health Aging. 2014;18(3):277-83.

9. Woo J, Leung J, Morley John E. Validating the SARC-F: a suitable community screening tool for sarcopenia? J Am Med Dir Assoc. 2014;15(9):630-4.

10. Miller J, et al. Validated screening tools for the assessment of cachexia, sarcopenia, and malnutrition: a systematic review. Am J Clin Nutr. 2018; 108(6):1196-208.

11. Ryoko K, et al. Calf circumference as a surrogate marker of muscle mass for diagnosing sarcopenia in Japanese men and women. Geriatr Gerontol Int. 2015;15(8):969-76

12. Rolland $Y$, Valérie Lauwers-Cances, Maxime Cournot, et al. Sarcopenia, calf circumference, and physical function of elderly women: a cross-sectional study. J Am Geriatr Soc. 2003;51(8):1120-1124.

13. Li Min S, Jiqi R, Haiyan, et al. The application of Ishii's scores in the screening for sarcopenia in community elderly. China Nurs Manage. 2018; 018(008):1034-8.

14. Florkowski CM, Sensitivity. Specificity, Receiver-Operating Characteristic (ROC) curves and likelihood ratios: communicating the performance of diagnostic tests. Clin Biochem Rev. 2008;29(Suppl 1):83-7.

\section{Publisher's Note}

Springer Nature remains neutral with regard to jurisdictional claims in published maps and institutional affiliations.
Ready to submit your research? Choose BMC and benefit from:

- fast, convenient online submission

- thorough peer review by experienced researchers in your field

- rapid publication on acceptance

- support for research data, including large and complex data types

- gold Open Access which fosters wider collaboration and increased citations

- maximum visibility for your research: over $100 \mathrm{M}$ website views per year

At BMC, research is always in progress.

Learn more biomedcentral.com/submissions 